\title{
Evolutionary cell biology
}

\section{P. Nick}

Published online: 3 March 2009

(C) Springer-Verlag 2009

The exemplary study of so-called model organisms has advanced our current knowledge of molecular players and mechanisms in cell biology. The advantages of model organisms are evident - the focus of many researchers on these models has released tremendous synergy and has led to the generation of genomic information, mutant collections, expression profiles, and numerous additional tools that are now available to assign gene functions. The comparison of different model organisms has uncovered evolutionary conserved patterns of molecular interactions, but it has also identified fundamental differences between model organisms from different taxa. To understand these differences is important because they are related to the specific characteristics of the respective organism. When one wants to understand something which is specific but too different to be derived easily from that what is known, one has to follow the path that has led to this final state. In other words, it is time to combine cell biology with evolution. The menu of models has to be extended by species that are interesting in terms of comparative evolution. Two contributions in the present issue exemplify this approach of an evolutionary cell biology:

\section{Evolution of organelle targeting}

The work by Shinozaki et al. (in this issue, doi:10.1007/ s00709-009-0031-1) investigates the peroxisomal targeting signals PTS1 and PTS2 in the Rhodophyte Cyanidiochyzon merolae. These signals are conserved between mammalian, yeast, and higher plant cells. However, the PTS2 signal seems to be absent from this primitive red algae. In order to understand when these signals evolved, the authors searched for homologues of the plant peroxisomal targeting signal in the green algae Chlamydomonas reinhardtii. By fusion to a

P. Nick $(\bowtie)$

Institute of Biology, University Karlsruhe,

Kaiserstrasse 2,

76128 Karlsruhe, Germany

e-mail: peter.nick@bio.uni-karlsruhe.de modified form of the green fluorescent protein as reporter, they can show that these targeting signals are functional in the chlorophyte Closterium ehrenbergii that has large cells and therefore is well suited for localization studies. The granular structures visualized by the reporter match with the signals for reactive oxygen species, confirming the peroxisomal nature of the granulae. They arrive at the conclusion that the peroxisomal targeting machinery in the Chlorophyta is closely related to that present in higher plants, whereas in the Rhodophyte $C$. merolae, only the so-called PTS1 machinery is present, resembling a situation that is also found in Caenorhabditis elegans and supporting a model where ancient protista lack the PTS2 type of peroxisomal transport.

\section{Evolution of the mitotic spindle}

The work by Pickett-Heaps and Forer (in this issue, doi:10.1007/s00709-009-0030-2) adopts an explicitly evolutionary viewpoint on anaphase $\mathrm{A}$ - the mitotic phase where the chromosomes move polewards. They argue that it is very unlikely that cells evolved completely new and separate motility systems for mitosis, but rather redefined components that were already present in the interphase cell. This would explain why not only microtubules but also the actomyosin motility system participates in mitosis. This so-called matrix model proposes that actomyosin in the spindle matrix interacts with microtubules during anaphase $\mathrm{A}$. The role of the kinetochore fibers is reassessed towards a model where the fibers act to slow down the poleward movement of the chromosomes such that they can be correctly attached to the spindle. They illustrate their model by using the diatom spindle as case study and propose an evolutionary track initiating from a cytoplast organized around a radial microtubule cytoskeleton that was divided by a spindle of overlapping microtubules. Since cytokinesis in unwalled cells was actinbased cleavage, the modern spindle might have evolved by linking both motility systems - the resulting functional redundancy would provide a "safety net" for this highly sensitive and essential step of the cellular life cycle. 